\section{Kanert-Preis nach Bochum}

Die im Stifterverband beheimatete Stiftung Kanert für Allergieforschung hat ihren mit $10.000 €$ dotierten Förderpreis dieses Jahr an Priv.-Doz. Dr. Monika Raulf-Heimsoth und Dr. Hans-Peter Rihs aus Bochum vergeben.

D ie beiden Preisträger vom Berufsgenossenschaftlichen Forschungsinstitut für Arbeitsmedizin an der Ruhr-Universität Bochum sind molekularen Zusammenhängen zwischen dem gleichzeitigen Vorliegen einer $\mathrm{Na}$ turlatexallergie und einer Nahrungsmittelallergie auf der Spur. Besonders häufige Auslöser solcher Kreuzreaktionen sind Früchte wie Banane, Kiwi, Avocado, Tomate, Paprika oder Esskastanie. Daneben kommen bei Naturla- texallergie aber auch Reaktionen gegen Allergene von Beifuß, Birke oder Ficus benjamina vor.

Die Stiftung Kanert für Allergieforschung wurde 1993 vom Kölner Finanzexperten Horst Kanert errichtet. Sie unterstützt Forschungsvorhaben und vergibt Stipendien im Bereich der allergologischen Forschung. Die Verleihung des Förderpreises erfolgte dieses Jahr im Rahmen des 19. Fortbildungskongresses „Fortschritte der Allergolo-

\title{
Preis für engagierte Pädiaterin
}

Im Rahmen ihrer 6. Jahrestagung im August in Dresden verlieh die Gesellschaft für Pädiatrische Allergologie und Umweltmedizin (GPA) erstmals den mit $5.000 €$ dotierten „Förderpreis Pädiatrische Allergologie“. Ausgezeichnet wurde Dr. Sabine Schmidt von der gemeinnützigen Kinderumwelt GmbH in Osnabrück.

D er von Pharmacia Diagnostics gestiftete „Förderpreis Pädiatrische Allergologie " honoriert Veröffentlichungen oder Projekte, die in besonderer Weise zur Verbesserung der Situation von allergiekranken Kindern und Jugendlichen beitragen. Über die Vergabe entscheidet ein Kuratorium aus wissenschaftlich tätigen Kinderallergologen und Vertretern der GPA sowie des Sponsors.

Dr. med. Sabine Schmidt, die diesjährige Preisträgerin, wurde für ihre engagierte Arbeit bei der gemeinnützigen Kinderumwelt $\mathrm{GmbH}$ ausgezeichnet. Die Fachärz- tin für Kinderheilkunde und Allergologin gehört mit Prof. Dr. Karl Ernst von Mühlendahl zu den Urhebern dieser Dokumentations- und Informations-

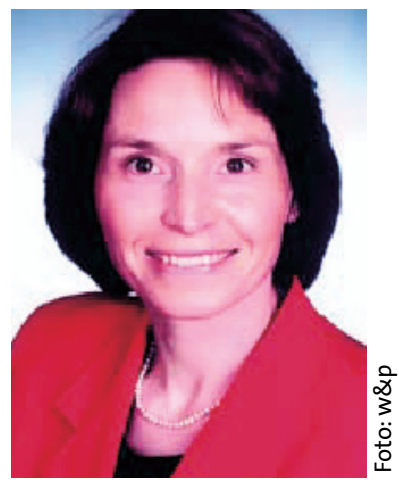

Dr. Sabine Schmidt, Preisträgerin des „Förderpreis Pädiatrische Allergologie“ 2003 stelle für Allergiefragen im Kindesalter. Hier entwickelte Dr. Schmidt auch die in Deutschland einzigartige Internetplattform uminfo - adiz - allinfo, die es Kinderärzten, Allergologen und Umweltmedizinern ermöglicht, schnell auf qualitätsgesicherte allergologische Informationen zuzugreifen.

In seiner Laudatio würdigte der erste Vorsitzende der GPA, Dr. Ernst Rietschel, Köln,

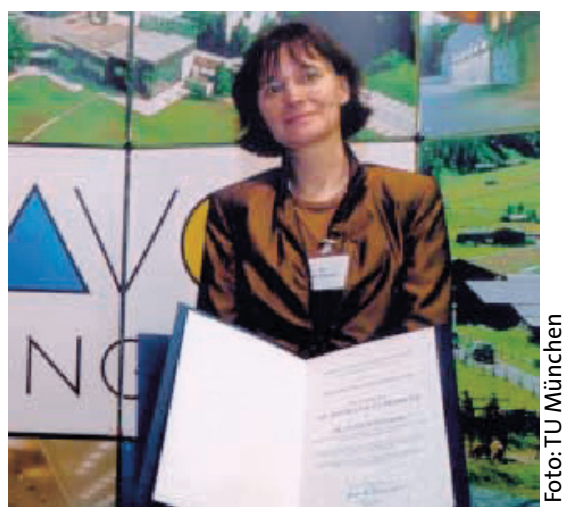

Priv.-Doz. Dr. Monika Raulf-Heimsoth präsentiert in Davos die Verleihungsurkunde des Förderpreises der Stiftung Kanert für Allergieforschung

gie, Dermatologie und Immunologie“ im September in Davos. Insgesamt wurde die Auszeichnung damit zum vierten Mal vergeben. red

Schmidts hervorragende Sachkenntnis und ihr schnelles ergebnisorientiertes Arbeiten. Fachverbände wie die GPA griffen deshalb auch gerne auf diese Kompetenzen zurück: So seien wesentliche Positionspapiere und Leitlinien der GPA ebenso Schmidts Feder entsprungen wie die Informationen der Internetplattform Pädinform und die Ratschläge des Präventions- und Informationsnetzwerks Allergie/Asthma (pina).

Kandidaten für den „Förderpreis Pädiatrische Allergologie“" 2004 können noch bis zum 1. Februar 2004 bei der Geschäftsstelle der GPA, Rathausstr. 10, 52072 Aachen, vorgeschlagen werden.

red

\section{Deutsche Präsidentschaft}

Im Rahmen des World Allergy Congress 2003 im September in Vancouver wurde Prof. Dr. med. Dr. phil. Johannes Ring, Direktor der Klinik und Poliklinik für Dermatologie und Allergologie am Biederstein der Technischen Universität München, erneut in den Vorstand der World Allergy Organization (WAO) gewählt. Gleichzeitig wurde Prof. Ring zum Kongresspräsidenten des nächsten World Allergy Congress der WAO in München im Sommer 2005 bestimmt. 